\title{
Standardising measures in pediatric rheumatology: the experience of the international consensus dataset for juvenile dermatomyositis
}

\author{
Giovanni Filocamo \\ Fondazione IRCCS Cà Granda Ospedale Maggiore Policlinico, Milan, Italy \\ Correspondence to: Giovanni Filocamo, MD, PhD. Reumatologia Pediatrica, UOC Pediatria a Media Intensità di Cure, Clinica de Marchi, Fondazione \\ IRCCS Cà Granda Ospedale Maggiore Policlinico, Via della Commenda 9, Milano 20124, Italy. Email: giovanni.filocamo@policlinico.mi.it. \\ Comment on: McCann LJ, Pilkington CA, Huber AM, et al. Development of a consensus core dataset in juvenile dermatomyositis for clinical use to \\ inform research. Ann Rheum Dis 2018;77:241-50.
}

Submitted Sep 03, 2018. Accepted for publication Sep 13, 2018.

doi: $10.21037 / \mathrm{atm} .2018 .09 .43$

View this article at: http://dx.doi.org/10.21037/atm.2018.09.43

In 1883 , the Scots-Irish mathematical physicist and engineer usually known as Lord Kelvin wrote "When you can measure what you are speaking about and express it in numbers, you know something about it; but when you cannot measure it, when you cannot express it in numbers, your knowledge is of a meagre and unsatisfactory kind" (1). There is no single measure for assessing children with rheumatic diseases, which is why clinical researchers use means of simultaneously quantifying multiple disease domains. However, although it is being increasingly acknowledged that the quality of care could be improved by the routine use of quantitative evaluations (2), these have rarely been adopted in everyday clinical practice by pediatric rheumatologists.

The fact that current laboratory tests and radiological examinations are insufficient to recognise changes in clinical phenotypes (3) has led international societies of pediatric rheumatology to develop and validate standardised outcome measures that can be used to provide information about disease activity, therapeutic responses, disease-related joint damage and disease remission. Furthermore, the complexity of assessing rheumatic diseases has also led to the introduction of composite indices, measures and scales.

The most useful means of developing new scales is based on creativity. The use of Delphi (4) or nominal group (5) techniques is an effective way of arriving at the consensus that is often required in order to ensure the international acceptance of clinical and research outcome measures. The former is essentially a series of questionnaires of which the first asks respondents to answer broad questions, and the answers are then used to build the subsequent questionnaires in a process that comes to an end once consensus has been reached. The nominal group technique is another a structured form of brainstorming that is intended to ensure the equal participation of group members in generating information that should ultimately lead to a satisfactory conclusion to the discussion of the issue(s) in question. Consensus conferences may be preceded by a series of Delphi questionnaires as a means of adding greater focus to nominal group tasks (3).

Pediatric rheumatologists are now paying Increasing attention to parent- and child-reported outcomes (PCROs) (6), which have the advantage of capturing parents' and children's perceptions of disease status and therapeutic results, and can lead to improvements in the quality of care (7). Including patients' and parents' perspectives in clinical assessments can not only make a significant contribution to medical decision-making, but also and increase the probability of successful treatment as it may well improve compliance to the prescribed therapy (7).

\section{Juvenile dermatomyositis (JDM)}

JDM is a rare, multi-system inflammatory disease of unknown etiology that mainly affects the skin and skeletal muscles, but may also affect visceral organs and give rise to sometimes serious complications (8). International collaboration is a crucial means of improving our 
understanding of this rare disease, and can be facilitated by the creation of user-friendly, web-based, accessible diseasespecific registries that can contribute to epidemiological research, reveal previously unknown risk factors, identify appropriate clinical trial participants, and raise the standard of care by encouraging the monitoring of major variables. The construction of such databases can help to define disease outcomes and inform therapeutic decision making, although care must be taken to ensure that they do not become unwieldy or require too much effort and time to keep them updated (9).

As there were once only a few widely used but unvalidated means of measuring outcomes in patients with myositis, the assessment of outcomes in therapeutic trials was simply based on non-standardised measurements of muscle strength and function. However, the collaborative International Myositis Assessment and Clinical Studies (IMACS) group developed a core set of outcome measures that take into account the fact the clinical and laboratory manifestations of dermatomyositis may be due to active inflammation or organ damage and were designed to assess myositis disease activity, disease-related damage, and the patients' health-related quality of life in clinical trials (10).

Furthermore, over the last 10 years, core sets for measuring outcomes in JDM have been developed by the IMACS group in America $(11,12)$ and the Paediatric Rheumatology International Trials Organisation (PRINTO) in Europe $(13,14)$. However, these had the limitations that, although their criteria often overlapped, there were some important differences, and they were not internationally agreed; in addition, as they were primarily designed for use in clinical trials, they were not necessarily so useful in everyday practice (15).

In order to overcome these limitations, the two organisations came together to develop a new set of consensus-driven response criteria for adults with dermatomyositis/polymyositis and children with JDM that allow disease improvement to be quantitatively measured and bridge the differences between PRINTO and IMACS (16). This took the form of a study aimed at establishing a JDM dataset covering disease sub-phenotypes, activity, damage over time and co-morbidities (17) that involved a large group of specialists in myositis (physicians and clinical scientists working in the fields of rheumatology, neurology or dermatology), as well as patients and parents.

The declared aim of the study was to find international consensus on a minimal practical dataset of clinical, laboratory and patient/parent-reported measures that included all of the variables necessary to make a standardised assessment of disease activity and monitor any changes. The study's Steering Committee first developed an initial dataset on the basis of existing international juvenileand adult-onset myositis databases; this was then submitted to a two-stage Delphi process followed by a consensus meeting in order to define a consensus core dataset, which was tested in clinical practice.

The proposed dataset consisted of 123 items divided into three sections: section A included 12 items (plus an additional six depending on the responses to them) designed to be completed once, at the time of the first data entry; section B consisted of 56 items (plus an additional 20 depending on the responses to them) designed to be completed at the time of every follow-up examination and representing each patient's current; and section $\mathrm{C}$ consisted of 55 items (plus an additional 15 depending on the responses to them) designed to be completed at baseline and every year thereafter in order to reflect the progression of disease-related damage.

The questionnaires were reviewed by the coordinators of patient and public advocacy groups and by focus groups of parents and children, with the latter also reviewing the patient/parent-reported outcome measures (PROMs) used for JDM and other rheumatological conditions. The patient/parent questionnaires consisted of 30 items: 23 from the Delphi adaptation (which combined or simplified items from the questionnaire submitted to healthcare professionals that would be of particular interest to patients/ parents), two added by the Steering Committee in order to investigate patient/parent attitudes to the collection and storage of information, and five suggested by the focus groups of patients and parents. Responses were obtained from Europe and North America, but other countries were under-represented (3\%).

This thorough process of establishing international consensus allowed the formulation of a consensus JDM dataset. However (and as pointed out by the study's authors themselves), even when the results of the Delphi process, the surveys of the patients/parents and suggestions from the UK patient focus group were taken into account, it was not possible to establish internationally agreed PROMs of function (activity) or health-related quality of life (HRQoL). Nevertheless, this difficulty was discussed and recorded.

It is also worth mentioning that it was decided to keep the functional tests of both the IMACS and PRINTO measures of muscle strength [manual muscle testing (MMT)] and the Childhood Myositis Assessment Scale 
(CMAS) in the consensus dataset, may be considered redundant in what was intended to be a minimal practical core dataset that was feasible for use in everyday clinical practice. However, a Hybrid Measure of Muscle Strength in JDM has recently be developed on the basis of 8-muscle MMT and the CMAS that is more comprehensive than the former and more feasible than the latter (8).

The consensus meeting also concluded that future development priorities were a means of assessing skin that can be more feasibly used in busy clinical settings, a shortened means of muscle assessment that excludes the redundancy implicit in combining CMAS and MMT, and patient-acceptable PROMs applicable to JDM.

The study described above represents an excellent example of international collaboration aimed at developing a universally accepted dataset by means of rigorous methodology that is capable of overcome the limitations arising from the use of different disease assessment measures in different countries have often made it difficult to compare data and organise transoceanic research.

\section{Acknowledgements}

None.

\section{Footnote}

Conflicts of Interest: The author has no conflicts of interest to declare.

\section{References}

1. Buchanan WW, Smythe HA. Can clinicians and statisticians be friends? J Rheumatol 1982;9:653-4.

2. Filocamo G, Consolaro A, Solari N, et al. Recent Advances in Quantitative Assessment of Juvenile Idiopathic Arthritis. Ann Paediatr Rheum 2012;1:84-96.

3. Brunner HI, Ravelli A. Developing outcome measures for paediatric rheumatic diseases. Best Pract Res Clin Rheumatol 2009;23:609-24.

4. Dalkey NC, Brown BB, Cochran S. The Delphi method an experimental study of group opinion (microform:). Santa Monica, CA: Rand Corp, 1969.

5. Delbecq AL. "Matrix organization"; a conceptual guide to organizational variation. Madison: University of Wisconsin, Bureau of Business Research and Service, 1969.

6. Consolaro A, Giancane G, Schiappapietra B, et al. Clinical outcome measures in juvenile idiopathic arthritis. Pediatr
Rheumatol Online J 2016;14:23.

7. Berard R, Laxer RM. Improving the quality of care in children with juvenile idiopathic arthritis: a step in the right direction. J Rheumatol 2011;38:789-90.

8. Varnier GC, Rosina S, Ferrari C, et al. Development and Testing of a Hybrid Measure of Muscle Strength in Juvenile Dermatomyositis for Use in Routine Care. Arthritis Care Res (Hoboken) 2018;70:1312-9.

9. McCann LJ, Arnold K, Pilkington CA, et al. Developing a provisional, international Minimal Dataset for Juvenile Dermatomyositis: for use in clinical practice to inform research. Pediatr Rheumatol Online J 2014;12:31.

10. Miller FW, Rider LG, Chung YL, et al. Proposed preliminary core set measures for disease outcome assessment in adult and juvenile idiopathic inflammatory myopathies. Rheumatology (Oxford) 2001;40:1262-73.

11. Rider LG, Giannini EH, Harris-Love M, et al. Defining Clinical Improvement in Adult and Juvenile Myositis. J Rheumatol 2003;30:603-17.

12. Rider LG, Aggarwal R, Pistorio A, et al. 2016 American College of Rheumatology/European League Against Rheumatism Criteria for Minimal, Moderate, and Major Clinical Response in Juvenile Dermatomyositis: An International Myositis Assessment and Clinical Studies Group/Paediatric Rheumatology International Trials Organisation Collaborative Initiative. Ann Rheum Dis 2017;76:782-91.

13. Ruperto N, Ravelli A, Murray KJ, et al. Preliminary core sets of measures for disease activity and damage assessment in juvenile systemic lupus erythematosus and juvenile dermatomyositis. Rheumatology (Oxford) 2003;42:1452-9.

14. Ruperto N, Pistorio A, Ravelli A, et al. The Paediatric Rheumatology International Trials Organisation provisional criteria for the evaluation of response to therapy in juvenile dermatomyositis. Arthritis Care Res (Hoboken) 2010;62:1533-41.

15. Rider LG, Werth VP, Huber AM, et al. Measures of adult and juvenile dermatomyositis, polymyositis, and inclusion body myositis: Physician and Patient/Parent Global Activity, Manual Muscle Testing (MMT), Health Assessment Questionnaire (HAQ)/Childhood Health Assessment Questionnaire (C-HAQ), Childhood Myositis Assessment Scale (CMAS), Myositis Disease Activity Assessment Tool (MDAAT), Disease Activity Score (DAS), Short Form 36 (SF-36), Child Health Questionnaire (CHQ), physician global damage, Myositis Damage Index (MDI), Quantitative Muscle Testing (QMT), Myositis Functional Index-2 (FI-2), Myositis Activities Profile 
(MAP), Inclusion Body Myositis Functional Rating Scale (IBMFRS), Cutaneous Dermatomyositis Disease Area and Severity Index (CDASI), Cutaneous Assessment Tool (CAT), Dermatomyositis Skin Severity Index (DSSI), Skindex, and Dermatology Life Quality Index (DLQI). Arthritis Care Res (Hoboken) 2011;63 Suppl 11:S118-57. 16. Varnier GC, Pilkington CA, Wedderburn LR. Juvenile

Cite this article as: Filocamo G. Standardising measures in pediatric rheumatology: the experience of the international consensus dataset for juvenile dermatomyositis. Ann Transl Med 2018;6(Suppl 1):S33. doi: 10.21037/atm.2018.09.43 dermatomyositis: novel treatment approaches and outcomes. Curr Opin Rheumatol 2018;30:650-4.

17. McCann LJ, Pilkington CA, Huber AM, et al. Development of a consensus core dataset in juvenile dermatomyositis for clinical use to inform research. Ann Rheum Dis 2018;77:241-50. 